Gynäkologe 2010 • [jvn]:[afp]-[alp] DOI 10.1007/s00129-010-2555-z

Online publiziert: 10. Juli 2010

(c) Springer-Verlag 2010

\author{
K. Diedrich · C. Anthuber · T. Dimpfl • D. Finas \\ Klinik für Frauenheilkunde und Geburtshilfe, \\ Universitätsklinikum Schleswig-Holstein Campus Lübeck
}

\section{Grenzsituationen in der Urogynäkologie}

"Morbiditätskarte", die der Erfassung aller Begleiterscheinungen und -erkrankungen und damit der Übersichtlichkeit dient.

Im Mittelpunkt des Beitrags von Keim et al. steht die Behandlung der jungen $\mathrm{Pa}-$ tientin. Diese stellt eine besondere Herausforderung dar, da insbesondere die prospektive Lebenserwartung und die Familienplanung in das therapeutische Konzept einbezogen werden müssen. Im Hinblick auf die Lebensqualität sollte, wenn es opportun erscheint, ein Stufenschema mit zunächst konservativem und wenn erforderlich dann operativem Vorgehen gewählt werden. Die Wahl des operativen Verfahrens sollte altersangepasst unter Berücksichtigung möglicher Komplikationen und Einschränkungen erfolgen.

Im Fokus des Beitrags von Dimpfl et al. steht die ältere Patientin. Es werden für dieses Kollektiv konservative und geeignete operative Verfahren ausgewählt, dargestellt und verglichen. Dieses Patientengut bringt zur Problematik der Inkontinenz bzw. Beckenbodeninsuffizienz weitere Morbiditäten mit. Daher werden zur Minimierung des operativen Risikos grundsätzliche Gedanken zur präoperativen Diagnostik und dem perioperativen Management thematisiert.

Der Beitrag von Finas et al. wendet sich dem Kollektiv der onkologischen Patientinnen zu, einer wenig beachteten Gruppe von Patientinnen, die unter den Folgen der onkologischen Therapie oder z. T. aggravierten präexistenten Problemen leiden. Spezifische Aspekte der Prävention, der konservativen und operativen Therapie sowie der Interaktion mit der onkologischen Therapie der Patientinnen mit hohem Leidensdruck werden dargestellt und diskutiert.
Pharmakologische Interaktionen werden in dem Beitrag von Szych et al. beleuchtet. Es werden Hinweise zu vorteilhaftem Wechsel von Substanzgruppen gegeben und Wirkmechanismen erläutert.

Einen kurzen Überblick über die interdisziplinäre Zusammenarbeit und mögliche Diagnose- und Behandlungspfade gibt der Beitrag von Tunn. Hier werden Denkanstöße zur kooperativen Medizin im Grenzbereich der Disziplinen gegeben.

\section{( ) Beckenbodeninsuffi- zienz und Inkontinenz müssen interdisziplinär bearbeitet werden}

In einer intensiven Auseinandersetzung mit dem Einfluss von Eingriffen am Beckenboden auf die Sexualität geben Peschers et al. in ihrem Beitrag einen profunden Einblick in die Folgen unseres operativen Handelns und die Auswirkungen auf die Sexualität unserer Patientinnen und ihrer Partner.

Diagnostik und Therapie von Beckenbodeninsuffizienz und Inkontinenz müssen in einem interdisziplinären Zusammenhang erfolgen. Dabei ist darauf zu achten, dass prophylaktische operative Eingriffe nicht erfolgen sollten, sondern ausschließlich symptom-, befund- und erforderniskorrelierte nach offener, intensiver und gut dokumentierter Aufklärung. Es sind nicht nur anatomische und funktionelle, sondern auch forensische Erwägungen anzustellen. Eine weitere Verbesserung stellt die Dokumentation der Behandlungsverläufe und -ergebnisse in Registern sowie die Intensivierung der Forschung dar. Die Bildung von Kontinenz- 
oder Beckenbodenzentren dient auch mit Qualitätssicherung/-erhalt und Qualitätssteigerung diesen Zielen. Alle aktuell praktizierten Zertifizierungsverfahren der Fachgesellschaften von Gynäkologen, Urologen, Koloproktologen u. a. werden in Zukunft durch eine interdisziplinäre Zertifizierung mit einem gemeinsamen Zertifizierungsverfahren der drei großen wissenschaftlichen Fachgesellschaften von Gynäkologen, Urologen und Koloproktologen ersetzt.

Mit diesem Themenheft von Der Gynäkologe wollen wir die ärztliche Vigilanz erhöhen und einen relevanten Beitrag zu einem bedeutenden gesellschaftlichen Komplex leisten. In der Fokussierung auf Grenzsituationen zeigt sich, dass diese Grenzen zum Wohle unserer Patientinnen - auch gegen ökonomische Zwänge - dringend überschritten werden müssen.

Wir wünschen Ihnen eine anregende Lektüre.

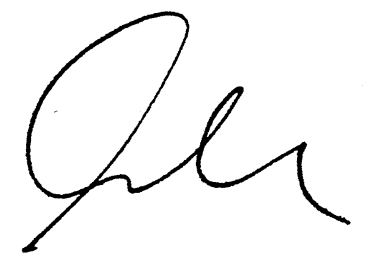

Prof. Dr. K. Diedrich

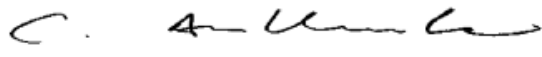

Prof. Dr. C. Anthuber

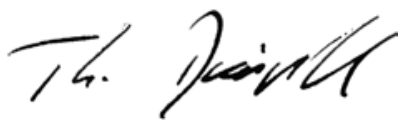

Prof. Dr. T. Dimpfl

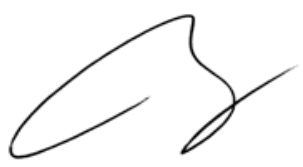

PD Dr. D. Finas

\section{Korrespondenzadresse}

\section{PD Dr. D. Finas}

Klinik für Frauenheilkunde und Geburtshilfe, Universitätsklinikum Schleswig-Holstein Campus Lübeck Ratzeburger Allee 160, 23538 Lübeck finas.d@arcor.de
Der Gynäkologe bietet jeden Monat umfassende und aktuelle Beiträge zu interessanten Themenschwerpunkten aus allen Bereichen der Gynäkologie und Geburtshilfe.

Möchten Sie ein bereits erschienenes Heft nachbestellen? Die bereits erschienenen Ausgaben aus 2009 und 2010 können Sie direkt bei unserem Kundenservice zum Preis von EUR 32,- beziehen:
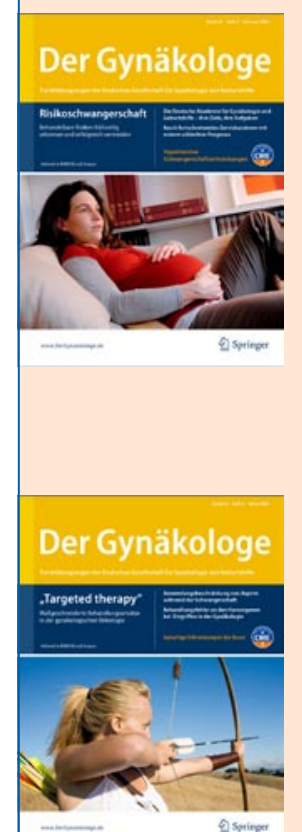

Leitthemen in Der Gynäkologe 2009

- 01/09 Habituelle Aborte

- 02/09 Risikoschwangerschaft

- 03/09 Targeted Therapy

- 04/09 Erkrankungen von Vulva und Vagina

- 05/09 40 Jahre Cardiotokographie

- 06/09 Neue Aspekte der Strahlentherapie in der Gynäkologie

- 07/09 Reproduktionsmedizin und Embryonenschutz

- 08/09 Verhalten in kritischen Rechtssituationen

- 09/09 Mammakarzinom in besonderen Situationen

- 10/09 Infektionserkrankung in Gynäkologie und Geburtshilfe

- 11/09 Prädiktive Diagnostik in der Frauenheilkunde

- 12/09 Zervixkarzinom

\section{Leitthemen in Der Gynäkologe 2010}

- 01/10 Verhalten in kritischen Rechtssituationen, Teil II

- 02/10 Innovationen in der minimal-invasiven Chirurgie der Gynäkologie

- 03/10 Zentrumsbildung in der Gynäkologie und Geburtshilfe

- 04/10 Dokumentationssysteme in der Frauenheilkunde

- 05/10 Hormontherapie in der Peri- und Postmenopause

- 06/10 Medizin am Rand der Lebensfähigkeit

- 07/10 Molekularbiologische Mechanismen für den Kliniker

- 08/10 Grenzsituationen in der Urogynäkologie

- 09/10 Frauenärztin/Frauenarzt der Zukunft

- 10/10 Psychosoziale Aspekte der Gynäkologie

- 11/10 Endometriose

- 12/10 Mammakarzinom - Neue Aspekte aus Praxis und Politik

(Änderungen vorbehalten)

So erreichen Sie unseren Kundenservice:

Springer Medizin Verlag GmbH

Kundenservice Zeitschriften

Haberstraße 7

69126 Heidelberg

Tel. +496221 345-4303

Fax: +49 6221 345-4229

E-Mail: Leserservice@springer.com

www.DerGynaekologe.de 